\title{
GEOMETRIA ED ELASTICITÀ: FOLDING DI UN SOLIDO, DRIPPING DI UN'ASTA ELASTICA E STRUTTURE SELF-OSCILLATING
}

\author{
DAVIDE BIGONI (*)
}

SuNTO. - Il filo conduttore dell'attività di ricerca descritta sono le condizioni in cui un solido o una struttura elastica soggetti a situazioni di rigidezza o spostamento estreme possono innescare e sviluppare figure deformative di geometria complessa. Si descrive dapprima il folding ed il faulting di un solido elastico tridimensionale (con struttura di Cosserat e rapporti di anisotropia molto spinti), poi il dripping di un'asta elastica (generato da forze configurazionali) ed infine la possibilità che una struttura elastica soggetta ad una sorgente uniforme di energia possa generare una oscillazione di frequenza costante, diventando così un sistema self-oscillating.

$* * *$

ABSTRACT. - The leitmotiv of the described research activity is the situation in which an elastic solid or an elastic structure can spontaneously generate complex geometrical patterns, when subjected to extreme deformation. In this context, folding and faulting are described for a three-dimensional elastic solid with extreme anisotropy. The dripping of an elastic rod is presented (as generated by configurational forces) and finally the possibility of a self-oscillating elastic system is introduced, where an elastic structure oscillates with a given frequency when subject to a steady energy input.

\section{INTRODUZIONE}

Quando l'anisotropia di un materiale elastico assume contrasti di rigidezza estremi o quando le deformazioni di una struttura avvengono

(*) Dipartimento di Ingegneria Civile, Ambientale e Meccanica, Università di Trento, Italia. E-mail: bigoni@ing.unitn.it 
nel campo nonlineare, si osserva spesso l'insorgenza di deformazioni che assumono forme geometriche complesse. Un esempio di questo comportamento è rappresentato dai modi a "forma di diamante" che si verificano nella instabilità di un cilindro sottile compresso e che si possono semplicemente visualizzare schiacciando una tubo sottile di gomma con le mani. Lo studio di questi problemi meccanici, in cui pattern deformativi complessi si sviluppano sotto carichi crescenti, può essere importante da diversi punti di vista, tra l'altro nella progettazione di materiali così detti "origami", capaci di cambiare forma e dimensioni a seconda delle funzionalità che devono assolvere, o delle strutture deployable, che possono essere sviluppate nello spazio a partire da un nucleo di piccole dimensioni. Sempre in questo ambito di ricerca è possibile formulare modelli meccanici per spiegare problemi di morfogenesi in biologia ed in geologia, ad esempio, per descrivere il processo di crescita delle circonvoluzioni del cervello, o le forme ondulate delle conchiglie marine, o il processo di modellazione dei rilievi della crosta terrestre.

Nel seguito si descrivono alcuni risultati ottenuti negli ultimi anni dal gruppo di Meccanica dei Solidi e delle Strutture dell'Università di Trento.

\section{FOLDING AND FAULTING DI UN CONTINUO ELASTICO}

Durante il processo di folding, la flessione di un elemento si localizza in una zona di ampiezza limitata, separata da zone che restano quasi indeformate. Questo processo si manifesta di rado in natura e non risulta facilmente inquadrabile nel modello dell'elasticità 'alla Cauchy'. Recentemente è stato mostrato che il folding può essere spiegato come una instabilità materiale, in particolare una perdita di ellitticità, nell'ambito dell'elasticità vincolata di Cosserat $[1,2,3]$.

Se si considera infatti un materiale alla Cosserat dotato di una ortotropia estrema, ovvero caratterizzato da un rapporto molto elevato tra rigidezze elastiche in direzioni diverse, si può dimostrare che il folding avviene spontaneamente come risposta ad una agente perturbativo quando il materiale è vicino al bordo ellittico. La tecnica perturbativa basata sulla funzione di Green, proposta per analizzare la risposta di un solido deformato fino alla prossimità di una instabilità materiale [4], mette in evidenza folding a piega singola al bordo ellittico/parabolico e a piega cruciforme al bordo ellittico/iperbolico. 
Quando un materiale di Cosserat con anisotropia spinta fino al raggiungimento del bordo ellittico viene perturbato con una coppia concentrata, si evidenzia la formazione di faulting, ovvero di una discontinuità del primo ordine negli spostamenti, caratterizzata da una ampiezza limitata e da una geometria a discontinuità singola (al bordo ellittico/parabolico) o a croce (al bordo ellittico/iperbolico).

\section{DRIPPING DI UN'ASTA ELASTICA}

E' stato mostrato come strutture elastiche soggette a grandi spostamenti possono mettere in luce forze configurazionali analoghe a quelle che agiscono sulle dislocazioni nei solidi $[5,6]$. L'esistenza di tali forze può essere semplicemente ricavata considerando una asta rettilinea elastica innestata ad un estremo in un manicotto privo di attrito e soggetta all'altro estremo ad una coppia concentrata. In queste condizioni si è portati a pensare che il manicotto reagisca semplicemente con una coppia uguale ed opposta a quella applicata. Se però si considera l'energia potenziale totale del sistema e si riconosce che la luce della asta può variare poiché il manicotto lascia libera la traslazione dell'asta, una derivata (cambiata di segno) di codesta energia, risulta in una forza configurazionale orizzontale (proporzionale al quadrato del momento applicato) che "sfila" l'asta dal manicotto.

L'esistenza di queste forze configurazionali, o "alla Eshelby", ha una profonda influenza sul comportamento di una classe ampia di strutture [7-9]; inoltre, facendo ricorso a tali forze è possibile ottenere la self-encapsulation di una asta, in cui quest'ultima, soggetta ad un carico trasversale, assume spontaneamente la forma di una goccia [10]. Il fatto che una asta elastica possa assumere la forma di una goccia non deve sorprendere poiché l'equazione della elastica di Eulero governa anche la forma della superficie di un menisco d'acqua in un canale capillare [11].

\section{Strutture SELF-OSCILLATING}

Strutture elastiche self-oscillating raggiungono dopo un breve transitorio un ciclo limite ad ampiezza e frequenza costante, quando sono soggette ad un input di energia uniforme nel tempo. Un esempio 
di queste strutture è rappresentato dal pendolo di Ziegler, dall'asta di Beck, due casi ben noti in meccanica strutturale. Si è mostrato come la forza follower necessaria a sviluppare il comportamento self-oscillating del pendolo di Ziegler e dell'asta di Beck può essere trasmessa alla struttura tramite un particolare vincolo attritivo alla Coulomb [12].

\section{RINGRAZIAMENTI}

Si ringrazia il finanziamento europeo FP7-PEOPLE-IDEASERC-2013-AdG (2014-2019) 'Instabilities and nonlocal multiscale modelling of materials'.

\section{BIBLIOGRAFIA}

Bigoni D, Gourgiotis PA, Folding and faulting of an elastic continuum. Proc. Royal Soc. A, 2016: 472: 20160018.

Gourgiotis PA, Bigoni D, Stress channelling in extreme couple-stress materials Part I: Strong ellipticity, wave propagation, ellipticity, and discontinuity relations. J. Mech. Phys. Solids, 2016: 88: 150-168.

Gourgiotis PA, Bigoni D, Stress channelling in extreme couple-stress materials Part I: Strong ellipticity, wave propagation, ellipticity, and discontinuity relations. J. Mech. Phys. Solids, 2016: 88: 169-185.

Bigoni D, Nonlinear Solid Mechanics, Cambridge University Press, Cambridge, 2012.

Bigoni D, Dal Corso F, Bosi F, Misseroni D, Eshelby-like forces acting on elastic structures: theoretical and experimental proof. Mech. Materials, 2015: 80: 368-374.

Bigoni D, Dal Corso F, Misseroni D, Bosi F Torsional locomotion. Proc. Royal Soc. A, 2014 470, 20140599.

Bosi F, Misseroni D, Dal Corso F, Bigoni D, An elastica arm scale. Proc. Royal Soc. A, 2014: 470: 20140232.

Bigoni D, Bosi F, Dal Corso F, Misseroni, D, Instability of a penetrating blade. J. Mech. Phys. Solids, 2014: 64: 411-425.

Bosi F, Misseroni D, Dal Corso F, Bigoni D, Development of configurational forces during the injection of an elastic rod. Extreme Mechanics Letters, 2015: 4: 83-88.

Bosi F, Misseroni D, Dal Corso F, Bigoni D, Self-encapsulation, or the 'dripping' of an elastic rod. Proc. Royal Soc. A, 2015: 471: 20150195.

Zaccaria D, Bigoni D, Noselli G, Misseroni, D. Structures buckling under tensile dead load. Proc. Royal Soc. A, 2011: 467: 1686-1700.

D. Bigoni and G. Noselli Experimental evidence of flutter and divergence instabilities induced by dry friction. J. Mech. Phys. Solids, 2011: 59, 2208-2226. 